\begin{tabular}{|c|r|r|r|r|r|} 
Revista Duazary & ISSN: $1794-5992$ & Vol. 13 & No. 2 & $105-110$ & Julio - Diciembre de 2016 \\
\hline
\end{tabular}

\title{
CUIDADO DE ENFERMERÍA EN LA SALUD COMUNITARIA
}

\author{
NURSING CARE COMMUNITY HEALTH
}

TÍTULO CORTO: CUIDADO DE ENFERMERÍA EN LA SALUD COMUNITARIA

Diana Acosta-Salazar ${ }^{1}$, Patricia Lapeira-Panneflex ${ }^{2}$, Ediltrudis Ramos-De La Cruz ${ }^{3}$

Recibido en septiembre 07 de 2015

Aceptado en enero 19 de 2016

\section{RESUMEN}

El Proceso de Atención de Enfermería (PAE) es una herramienta sistemática que facilita la cientificidad de los cuidados en las prácticas comunitarias del profesional de enfermería, la aplicación del método científico en la práctica comunitaria, permite a la enfermería brindar cuidados de forma lógica, sistemática e integral, revaluando las intervenciones para alcanzar los resultados planteados. Se inició con la valoración de Patrones Funcionales de Marjory Gordon y luego en la etapa de diagnóstico y planificación, se interrelacionó con North american nursing diagnosis association (NANDA), Nursing interventions classification (NIC) y Nursing outcomes classification (NOC). Es un estudio descriptivo y prospectivo. Se efectuó un análisis por medio de la aplicación de métodos: escala de medición de los rasgos socio demográfico, estudio de sintomatologías para el descubrimiento prematuro de trastornos mentales en la comunidad y la valoración por patrones funcionales. El PAE incluye diagnósticos más frecuentes, criterios de Resultados, indicadores, intervenciones y actividades para manejar la problemática comunitaria. Se evidenció alteración en los Patrones: Adaptación y Tolerancia al Estrés, Autopercepción-Autoconcepto, Rol-Relaciones, Sueño y Descanso y Percepción y Manejo de la Salud. Un cuidado estandarizado con la interrelación NANDA-NICNOC permite brindar un cuidado holístico desde el punto de vista de la salud mental comunitaria con un grado de cientificidad que enmarca el quehacer profesional proyectando el cuidado individual, familiar y comunitario.

Palabras clave: Salud comunitaria; Salud mental; Proceso Atención Enfermería

\section{ABSTRACT}

Process Nursing Care (PAE) is a systematic tool that facilitates the scientificity of care in community practice nurse, the application of scientific method in community practice, allows nursing to provide care in logical, systematic and comprehensive reassessing interventions to achieve the proposed results. It began with the valuation of Marjory Gordon Functional Patterns and then at the stage of diagnosis and planning North American Nursing Diagnosis

1. Enfermera de la Universidad del Magdalena, Magister en Desarrollo Social de la Universidad del Norte. Estudiante de Doctorado En Enfermería - Universidad de Carabobo, Venezuela. Correo: dianis276@hotmail.com

2. Enfermera de la Universidad Popular del Cesar, Magíster en enfermería área de énfasis materno perinatal de la Universidad Nacional de Colombia. Especialista en desarrollo infantil. Profesora del Área Materno Infantil del programa de Enfermería de la Universidad del Magdalena. Estudiante de Doctorado En EnfermeríaUniversidad de Carabobo, Venezuela. Correo: patrilape0618@hotmail.com

3. Enfermera de la Universidad Industrial de Santander. Magister en enfermería área de énfasis cronicidad de la Universidad Nacional de Colombia. Docente de Planta de la Universidad del Magdalena. Estudiante de Doctorado En Enfermería- Universidad de Carabobo, Venezuela. Correo: ediramo06@yahoo.es 
Association (NANDA), Nursing Interventions Classification (NIC) and Nursing Outcomes Classification (NOC) is interrelate. It is a descriptive and prospective study. Diagnosis was made by applying the instruments measuring scale of the socio-demographic characteristics, symptom questionnaire for early detection of mental disorders in the community and appreciation for functional patterns. The PAE includes more frequent diagnoses, criteria outcomes, indicators, interventions and activities to manage community issues. alteration was evidenced in patterns: Adaptation and Stress Tolerance, Self-perception-Self-concept-, Role-Relationships, sleep and rest and Perception and Health Management. A standardized NANDA-NIC-NOC can provide inter care holistic care from the perspective of community mental health with a degree of scientific nature that frames the professional work projecting the individual, family and community care.

Keywords: Community Health; Mental Health; Nursing Care Process

\section{INTRODUCCIÓN}

$E^{1}$ PAE es un sistema integral que permite brindar cuidados de enfermería, donde se relacionan de manera cíclica y constante las cinco etapas: valoración, diagnóstico, planificación, ejecución y evaluación que se relacionan entre sí, estas etapas se estudian en el individuo, familia y comunidad, pero en la práctica se superponen al igual que en el método científico, metodológicamente ${ }^{1}$.

Para la valoración de enfermería, primera etapa del PAE, se adoptó la valoración de enfermería a través de los Patrones Funcionales de Marjory Gordon². En las etapas de diagnóstico y planificación se interrelacionan con NANDA, NOC, NIC, taxonomías que permiten a los profesionales de enfermería utilizar un lenguaje estandarizado que facilita la recolección sistemática de la información necesaria, con esto se espera: ¿Cuáles son los diagnósticos formulados?, ¿qué resultados se espera? y ¿qué intervenciones se realizan?

El enfermero tradicionalmente se ha caracterizado por ser cuidador profesional del paciente, la familia y la comunidad, sin embargo, son muchas las intervenciones que no traspasan el cuidado individual e impactan poco en el entorno familiar y social, en que muchas veces se encuentra la génesis del comportamiento del individuo ${ }^{3}$. Bajo este paradigma se enmarca como prioritario el cuidado en salud mental comunitario. Es decir, es integral. Por lo tanto, este trabajo tiene como fin contribuir a la promoción, el diagnóstico temprano y tratamiento oportuno de las alteraciones en la salud mental comunitaria, mediante la aplicación de instrumentos de valoración ${ }^{4}$.

El Proceso de Enfermería aplicado en una comunidad de Santa Marta inició con la valoración, aplicando el instrumento de Patrones Funcionales de Gordon². Evidenciándose alteraciones en cinco patrones funcionales: Adaptación-tolerancia al estrés, AutopercepciónAutoconcepto, Rol-Relaciones, Sueño-Descanso y Percepción y Manejo de la Salud. Por lo anterior, el objetivo de esta investigación es establecer el plan de cuidados estandarizado de enfermería en la Salud Mental Comunitaria utilizando la interrelación de las taxonomías NANDA/NOC/NIC.

\section{MATERIALES Y MÉTODOS}

Estudio descriptivo y prospectivo cualicuantitativo, durante el 2010 -2011. La Muestra fue de 474 adultos que representan 59\% de la población del barrio Cristo Rey, es un estudio descriptivo y prospectivo. Las personas que participaron en esta investigación fueron escogidas de una muestra estratificada proporcional tomada intencionalmente de la población. Se realizó diagnóstico inicial mediante la aplicación de los instrumentos: Escala de medición de las Características Sociodemográficas, Cuestionario de Síntomas Para Detección Precoz de Trastornos Mentales en la Comunidad y la Valoración por Patrones Funcionales de Marjorie Gordon, se trabajó la interrelación NANDA/NIC/NOC 5 . Los datos fueron procesados y analizados mediante estadística descriptiva (frecuencia, porcentajes y promedios) utilizando el paquete estadístico para las ciencias sociales SPSS versión 
17. Se aplicó a cada persona que hizo parte del estudio un formato de consentimiento informado el cual fue socializado y firmado, se les garantizó confidencialidad, dignidad, seguridad y bienestar a todos los participantes. Se propendió por la protección de los derechos y el bienestar, entre ellos el respeto a la dignidad humana, a la privacidad e intimidad, y los principios de beneficencia y justicia todo esto bajo la declaración de Helsinki ${ }^{6}$.

\section{Declaración de Aspectos Éticos}

Se tuvo en cuenta todas las consideraciones estipuladas en la resolución 008430 de 1993 del ministerio de salud, por la cual se establecen las normas científicas, técnicas y administrativas para la investigación en salud, donde prevaleció el criterio del respeto a su dignidad y la protección de sus derechos y bienestar (Articulo 5).

\section{RESULTADOS Y DISCUSIÓN}

Las mujeres representan el 59\% de la población adulta entre 16 y 59 años, no trabajan fuera del hogar y por lo tanto el oficio más frecuente es el de ama de casa. El barrio en su totalidad es de estrato socio-económico uno, el 37\% de la población no está afiliada al SGSSS, el 18\% realizó la secundaria completa, casi la mitad $48 \%$ de la población vive en unión libre, el 22\% realiza trabajo ocasional, menos de la mitad son familias nucleares, manifiestan que la mayor preocupación es la familia, el $28,57 \%$ se preocupa por su familia, el $19,04 \%$ se preocupa por la salud, un $19,04 \%$ se preocupa por el dinero, el $10,47 \%$ se preocupa por el trabajo, un $16,19 \%$ por los hijos, por último el 6,66\% se preocupa por otras cosas. Según el instrumento $\mathrm{SQR}^{7}$ "personas en riesgo de padecer depresión y ansiedad”: el 43,8\% manifestaron sentirse nerviosos, tensos o aburridos, el $41 \%$ se asusta con frecuencia, el $36,2 \%$ se sienten tristes y solos, el 22,8\% manifestaron que no pueden pensar con claridad, el $29,5 \%$ se cansa con facilidad, $21,9 \%$ tiene frecuentes dolores de cabeza, $20 \%$ llora con mucha frecuencia, $29,5 \%$ tiene dificultad para tomar decisiones, $18,1 \%$ se siente cansado todo el tiempo, $17,1 \%$ tienen dificultad para disfrutar de sus actividades diarias, $21,9 \%$ tiene sensaciones desagradables en su estómago, $23,9 \%$ duerme mal, $15,2 \%$ ha perdido el interés en las cosas, $17,1 \%$ tiene mal apetito, 14,3\% sufre de mala digestión, 6,6\% tiene dificultad para hacer su trabajo, $2,9 \%$ sufre de temblor en las manos, 6,6\% se siente incapaz de desempeñar un papel útil en la vida, $4,7 \%$ ha pensado en la posibilidad de acabar con su vida, 6,6\% se sienten inútiles.
De igual forma, se distingue como afectación mental, a aquello que se relaciona con problemas emocionales, cognitivos y comportamental con realidades simbólicas, elaboradas cultural e históricamente en la mismísima confluencia social y comunitaria ${ }^{8}$. Por lo anterior, un ser humano considerado con una enfermedad mental, no solo se relación con alguna alteración de la personalidad si no con su forma de relacionarse y comunicarse con su entorno.

Franco et $\mathrm{al}^{9}$ refieren en su protocolo de diagnósticos de enfermería en salud mental coincide con el hallazgo de los cinco patrones alterados de esta investigación, donde trabaja con los mismos diagnósticos, criterios de resultados, indicadores, intervenciones y actividades de enfermería.

\section{Patrón de adaptación y tolerancia al estrés}

El 68\% padecen de una gran tensión, ansiedad y estrés, $58 \%$ no saben cómo afrontar sus problemas, $55 \%$ sienten gran tristeza, el $20 \%$ presentan frustración, desesperanza e impotencia, destacándose las etiquetas diagnósticas: Diagnóstico de enfermería (1): Afrontamiento inefectivo relacionado con $(\mathrm{R} / \mathrm{C})$ inadecuada solución de problemas manifestado por (M/P) dificultad para tomar decisiones, Problemas Sociales, Agentes Estresantes y Frustración Criterios de Resultados: Afrontamiento de problemas(1) y tolerancia al estrés (2). Indicadores: Utiliza el apoyo social disponible (1) y refiere disminución de los síntomas del estrés (2) Intervenciones: Aumentar el Afrontamiento y Potenciación de la seguridad Actividades: Evaluar la capacidad para tomar decisiones, favorecer la expresión de sentimientos, ambiente de confianza, proporcionar apoyo emocional y familiar ${ }^{10}$. Diagnóstico de enfermería (2): Duelo R/C muerte de una persona significativa M/P Sufrimiento, culpa, dolor, desespero. Criterios de Resultados: Resolución de la Aflicción(1) y equilibrio emocional(2). Indicadores: Expresa sentimientos sobre la pérdida, verbaliza la aceptación de la pérdida (1), control de los impulsos (2), interés y energía por lo que le rodea (2) Intervenciones: Facilitar el duelo y apoyo emocional. Actividades: Fomentar la expresión de sentimientos acerca de la perdida, ayudar a identificar estrategias personales de resolución de problemas ${ }^{11}$, uso de mecanismos de defensa, realizar afirmaciones de apoyo y proporcional ayuda en la toma de decisiones.

\section{Patrón de Autopercepción- Autoconcepto}

El 76\% padecen de mal estado de ánimo, el $45 \%$ presentan alterada sus habilidades (cognitivas, afectiva 
o física), el $62 \%$ presentan inconformidad por la imagen corporal e identidad, el $35 \%$ presentan sentimientos de temor, preocupación y desesperanza por la vida, los diagnósticos destacados: Diagnóstico de enfermería (1): Baja autoestima situacional $\mathrm{R} / \mathrm{C}$ falta de reconocimiento o recompensa $\mathrm{M} / \mathrm{P}$ Expresiones de si mismo como incapaz de afrontar los acontecimientos, expresiones de tristeza y de no tener control sobre una situación. Criterios de resultado: Autoestima. Indicadores: Verbalizaciones de autoaceptación, nivel de confianza y comunicación abierta. Intervenciones: Potenciación de la Autoestima. Actividades: Animar a identificar sus virtudes, animar a evaluar su propia conducta, mostrar confianza para controlar una situación. Diagnóstico de enfermería (2): Desesperanza R/C percepción de amenaza para el auto concepto y estado de salud. Criterio de Resultado: Esperanza y autocontrol de la depresión. Indicadores: Expresiones de Confianza, de creencia en si mismo, de optimismo, de ganas de vivir y referir ausencia de manifestaciones físicas de depresión. Intervenciones: Dar esperanza. Actividades: Ayudar a identificar áreas de esperanza en la vida, diseñar y revisar metas relacionadas con el objeto de esperanza y ampliar el mecanismo de resolución de problemas ${ }^{11}$.

\section{Patrón Rol-Relaciones}

Se evidencia que el $34 \%$ presentan poco compromiso del rol, se han presentado casos de delincuencia común, homicidios y violaciones, el $47 \%$ consume sustancias psicoactivas, el $53 \%$ presentan malas relaciones interpersonales e intrafamiliares, poco comunicación para resolver problemas. Se evidencia las etiquetas diagnosticas: Diagnósticos de enfermería (1): Interrupción de los procesos familiares $\mathrm{R} / \mathrm{C}$ Crisis situacional M/P sistema de comunicaciones cerradas e inefectivas, falta de apoyo familiar y emocional, incapacidad para adaptarse a los cambios e insatisfacción de necesidades. Criterios de Resultados: Normalización de la familia y funcionamiento de la familia. Indicadores: Comunica la importancia de mantener las actividades normales, mantiene las rutinas habituales, satisface las necesidades físicas y Psicológicas de la familia, pasar tiempo en familia. Intervenciones: Fomentar la normalización familiar y estimulación de la integridad familiar. Actividades: Animar a la familia a mantener una red social y un sistema de apoyo habituales, escuchar a los miembros de la familia, mantener relaciones positivas y animar a la familia a mantener los hábitos, rituales y rutinas normales. Diagnósticos de enfermería (2): Deterioro de la interacción social $\mathrm{R} / \mathrm{C}$ barreras de comunicación y falta de habilidades para la solución de problemas M/P incapacidad para comunicar una sensación satisfactoria de implicación social. Criterios de Resultado: Habilidades de interacción social. Indicadores: Divulgación, receptividad, utilizar métodos de resolución de conflictos, mostrar sensibilidad y calidez. Intervenciones: Aumentar los sistemas de apoyo. Actividades: Determinar el grado de apoyo familiar, determinar las barreras que lo afectan, fomentar las relaciones y participaciones familiares.

\section{Patrón Sueño y Descanso}

Hay muchos establecimientos como bares, cantinas, estaderos que producen contaminación auditiva que interfiere con la calidad del sueño, el $67 \%$ presenta problemas para conciliar el sueño y $34 \%$ solo puede dormir de 5 horas. El diagnóstico común: Diagnóstico de enfermería: Deterioro del patrón del sueño R/C Ansiedad, estrés familiar, y percepción de vulnerabilidad a peligros M/P Pesadillas, dificultad para conciliar el sueño y problemas familiares. Criterios de Resultados: Sueño. Indicadores: Horas de sueño, calidad del sueño y duerme toda la noche. Intervenciones: Mejorar el sueño. Actividades: Determinar un esquema de sueño, eliminar situaciones estresantes, instruir sobre el uso de técnicas de relajación, regular los estímulos del ambiente y determinar el grado de apoyo familiar.

\section{Percepción Manejo De La Salud}

En la comunidad frecuentemente se realizan brigadas de salud pero el servicio en el puesto de salud lo califica el 47,61\% como muy regular, Así mismo el $47.3 \%$ indicadores para alcoholismo y el $38.7 \%$ indicador de consumo de alguna sustancia psicoactiva durante su vida, el diagnóstico fue mantenimiento ineficaz de la salud $\mathrm{R} / \mathrm{C}$ déficit de conocimiento acerca de la promoción de la salud, las características definitorias acciones insuficientes de promoción de la salud y prevención de la enfermedad ${ }^{11}$. Criterios de Resultados: Conocimiento: Control del consumo de sustancia y Control del riesgo: Consumo de Alcohol Indicadores: Consecuencias sociales del consumo, Identifica los factores de riesgo del abuso de alcohol y busca información sobre el consumo de sustancia psicoactivas Intervenciones: Educación para salud. Actividades: Centrarse en los beneficios de salud positivo inmediatos o a corto plazo para conductas de estilo de vida positivas.

Es claro y visible lo necesario e importante de la promoción de la salud mental de tal forma que permita 
alcanzar un estado de adecuado bienestar físico, mental y social, así mismo trabajar en acciones orientadas a promover la equidad social, a reducir la diferencias y asegurar la igualdad de oportunidades ${ }^{12,13}$.

Los cuidados de Enfermería relacionados con la salud mental no dependen de que se cuente con los recursos físicos, sino de la calidad de servicio y de la calidez humana. Esto implica que le profesional de enfermería debe bridar un cuidado humanizado e integral desde una perspectiva holística y una complejidad bio-psicosocial ${ }^{14}$. Así mismo, se necesitan profesionales y equipos receptivos y disponibles para implicarse en la necesidad del abordaje emocional ${ }^{15}$.

Un cuidado estandarizado con la interrelación NANDANIC-NOC permite brindar un cuidado holístico desde la perspectiva de la salud mental comunitaria con un grado de cientificidad que enmarca el quehacer profesional proyectando el cuidado individual, familiar y comunitario.

\section{CONCLUSIÓN}

La salud mental de la comunidad del barrio Cristo Rey mostró que los adultos presentan ansiedad fácilmente, y se quejan de que no pueden pensar con claridad, duermen mal y se consideran personas de riesgo para depresión o angustia, se encuentran alterados cinco patrones de los once establecidos por Gordon ${ }^{16-19}$.

Las circunstancias actuales de la realidad comunitaria en enfermería requieren fomentar la salud mental y prevenir la enfermedad mental, sustentándose en la interrelación NANDA, NIC, NOC que brinda las herramientas para la intervención con el bagaje científico que enmarca el quehacer, es necesario mantener el compromiso profesional que proyecte el cuidado individual, familiar y comunitario $0^{20-24}$.

\section{DECLARACIÓN SOBRE CONFLICTOS DE INTERÉS}

No se presentaron conflictos de interés en el desarrollo de la investigación.

\section{REFERENCIAS BIBLIOGRÁFICAS}

1. Ramos E. El proceso de enfermería: una guía metodológica en el ejercicio profesional. Universidad del Magdalena. Colombia; 2010.
2. Gordon M. Patrones funcionales |Internet|. Madrid: Consejo Gral. de Enfermería. |consultado 9 de abril de 2013|. Disponible en http://www.nipe.enfermundi.com/ futuretense_cs/ccurl/NIPE/pdf/patrones_funcionales_ mgordon.pdf

3. Alarcón A, Miranda G, Palacios N. Diagnóstico comunitario En Salud. Asentamiento Lagos de Occidente. Popayán; 2005

4. Argueta C, Flamenco N, Gonzáles J. Diagnóstico de las necesidades de salud mental comunitaria. noviembre, 2003.

5. Johnson M, Bulechek G. NANDA, NOC, and NIC linkages: Nursing diagnoses, outcomes \& interventions. $2^{a}$ ed. St.Louis: Mosby; 2005.

6. Colombia. Ministerio de Salud. Resolución No 008430. Por la cual se establecen las normas científicas, técnicas y administrativas para la investigación en salud. República de Colombia, (4 de octubre1993).

7. Gonzalez T, Ramos E. Detección de Signos y Síntomas de alteraciones en la salud mental de los habitantes del barrio ocho de diciembre Santa Marta. Duazary. 2004; 2(1): $92-100$

8. González T. El cuidado de enfermería en la salud mental del ciclo evolutivo humano. Santa Marta: Universidad del Magdalena; 2009

9. Franco A, Gonzalez E, Cano I, Domínguez I, Flores I, Salgado J, Et al. Planes de Cuidado Enfermería S.M, Servicio Extremeño de Salud; 2008.

10. Orozco M. Guía de intervención en enfermería basada en la evidencia. ISS; 1998.

11. Von H, Gómez J. Mapa de riesgos y recursos. Impresión Nobel impresores;1995.

12. Cari M. Proceso de enfermería por patrones salud mental. Perú; 2010.

13. Restrepo M. Promoción de la Salud Mental: Un marco para la formación y la acción. Revista Ciencias de la Salud. 2006;4: 86-193.

14. Larban J. El modelo comunitario de atención a la salud mental. Sociedad Española de Psiquiatría y Psicoterapia del Niño y del Adolescente. 2010; (49): 205-224.

15. ACISAM - Asociación de Capacitación e Investigación para la Salud Mental - Propuesta de Intervención Comunitaria en Comunidades Afectadas por Desastres. San Salvador, El Salvador; 2001.

16. Moorhead S, Johnson M, Swanson E. Clasificación de Resultados de Enfermería (NOC). Elsevier España; 2014.

17. Bulechek G, Butcher H, Dochterman J, Wagner C. Clasificación de Intervenciones de Enfermería (NIC). Elsevier España; 2014.

18. Carpenito L. Manual de Diagnóstico Enfermeros. Lippincott Williams\& Wilkins España; 2013.

19. Durán M. Aspectos Conceptuales del Cuidado de Enfermería y su relación con la Práctica y la Investigación; 2000. 
20. Roldán A, Fernández M. Proceso de atención de enfermería. Invest. educ. enferm. 1999; 17(2): 79-93.

21. Amaro M. El método científico de enfermería, el Proceso de Atención, y sus implicaciones éticas y bioéticas. Cubana de Enfermería. 2004; 20 (1): 1-1.

22. Lapeira-Panneflex P, Acosta Salazar D. Conocimientos y prácticas sobre enfermedades diarreicas y respiratorias entre madres de una institución de salud. Duazary. 2014; 11(1):36-240
23. Inuca $\mathrm{V}$, Icaza $\mathrm{L}$, Recalde $\mathrm{R}$, Cevallos $\mathrm{J}$, Romero $\mathrm{N}$. Percepción de la participación de parteras y sanadores tradicionales andinos ecuatorianos en un programa de formación en salud. Duazary. 2014; 11 (2): 131 - 13

24. Morrison M. Fundamentos de enfermería en salud mental. Fundamentos de enfermería en salud mental. 1999; -84-8174-349-6-61, 65.

Para citar este artículo: Acosta-Salazar D, Lapeira-Panneflex P, Ramos-De La Cruz E. Cuidado de enfermería en la salud comunitaria. Duazary. 2016 julio; 13 (2): 105 - 110 